\title{
OPEN Penetration of MeV electrons into the mesosphere accompanying pulsating aurorae
}

\author{
Y. Miyoshi ${ }^{1 凶}$, K. Hosokawa ${ }^{2}$, S. Kurita ${ }^{3}$, S.-I. Oyama ${ }^{1,4,5}$, Y. Ogawa ${ }^{4,16,17}$, S. Saito ${ }^{6}$, \\ I. Shinohara ${ }^{7}$, A. Kero ${ }^{8}$, E. Turunen ${ }^{8}$, P. T. Verronen ${ }^{8,9}$, S. Kasahara ${ }^{10}$, S. Yokota ${ }^{11}$, T. Mitani ${ }^{7}$, \\ T. Takashima ${ }^{7}$, N. Higashio ${ }^{7}$, Y. Kasahara ${ }^{12}$, S. Matsuda ${ }^{7}$, F. Tsuchiya ${ }^{13}$, A. Kumamoto ${ }^{13}$, \\ A. Matsuoka ${ }^{14}$, T. Hori ${ }^{1}$, K. Keika ${ }^{10}$, M. Shoji' ${ }^{1}$ M. Teramoto ${ }^{15}$, S. Imajo ${ }^{14}$, C. Jun ${ }^{1} \&$ \\ S. Nakamura ${ }^{1}$
}

Pulsating aurorae (PsA) are caused by the intermittent precipitations of magnetospheric electrons (energies of a few keV to a few tens of $\mathrm{keV}$ ) through wave-particle interactions, thereby depositing most of their energy at altitudes $\sim 100 \mathrm{~km}$. However, the maximum energy of precipitated electrons and its impacts on the atmosphere are unknown. Herein, we report unique observations by the European Incoherent Scatter (EISCAT) radar showing electron precipitations ranging from a few hundred keV to a few MeV during a PsA associated with a weak geomagnetic storm. Simultaneously, the Arase spacecraft has observed intense whistler-mode chorus waves at the conjugate location along magnetic field lines. A computer simulation based on the EISCAT observations shows immediate catalytic ozone depletion at the mesospheric altitudes. Since PsA occurs frequently, often in daily basis, and extends its impact over large MLT areas, we anticipate that the PsA possesses a significant forcing to the mesospheric ozone chemistry in high latitudes through high energy electron precipitations. Therefore, the generation of PsA results in the depletion of mesospheric ozone through high-energy electron precipitations caused by whistler-mode chorus waves, which are similar to the well-known effect due to solar energetic protons triggered by solar flares.

Visible light from aurorae is emitted by the excitation of neutral atmospheric components ${ }^{1}$. The precipitation of magnetospheric electrons and their acceleration along the magnetic field lines is the primary mechanism responsible for discrete aurorae, which are seen as curtain-like structures from the ground. On the other hand, diffuse aurorae, which may not be easily visible from the ground, are caused by the precipitation of charged particles (principally electrons) without field-aligned accelerations. It is assumed that the precipitation of diffuse auroral electrons is caused by pitch-angle scattering through plasma wave interactions in the magnetosphere. Typical diffuse aurora mainly results from precipitation of $\sim 1 \mathrm{keV}$ electrons ${ }^{2}$. Pulsating aurorae (PsA) are a relatively faint class of diffuse aurorae that exhibits quasi-periodic luminosity modulations ${ }^{3,4}$. Sounding rocket and lowaltitude spacecraft observations have shown that these modulations are associated with intermittent electron precipitation ranging from a few $\mathrm{keV}$ to a few tens of $\mathrm{keV}^{4-9}$.

${ }^{1}$ Institute for Space-Earth Environmental Research, Nagoya University, Nagoya 464-8601, Japan. ${ }^{2}$ Graduate School of Communication Engineering and Informatics, University of Electro-Communications, Chofu 182-8585, Japan. ${ }^{3}$ Research Institute for Sustainable Humanosphere, Kyoto University, Uji 611-0011, Japan. ${ }^{4}$ National Institute of Polar Research, Tachikawa 190-8518, Japan. ${ }^{5}$ University of Oulu, Pentti Kaiteran katu 1, Linnanmaa, Oulu, Finland. ${ }^{6}$ National Institute of Information and Communications Technology, Tokyo 184-8795, Japan. ${ }^{7}$ Japan Aerospace Exploration Agency (JAXA), Sagamihara 252-5210, Japan. ${ }^{8}$ Sodankylä Geophysical Observatory, University of Oulu, Sodankylä, Finland. ${ }^{9}$ Space and Earth Observation Centre, Finnish Meteorological Institute, Helsinki, Finland. ${ }^{10}$ Graduate School of Science, University of Tokyo, Tokyo 113-0033, Japan. ${ }^{11}$ Graduate School of Science, Osaka University, Toyonaka 560-0043, Japan. ${ }^{12}$ Graduate School of Natural Science and Technology, Kanazawa University, Kanazawa 920-1192, Japan. ${ }^{13}$ Graduate School of Science, Tohoku University, Sendai 980-8578, Japan. ${ }^{14}$ Graduate School of Science, Kyoto University, Kyoto 606-8502, Japan. ${ }^{15}$ Graduate School of Engineering, Kyushu Institute of Technology, Fukuoka 820-8501, Japan. ${ }^{16}$ The Graduate University for Advanced Studies, SOKENDAl, Hayama 240-0193, Japan. ${ }^{17}$ Joint Support-Center for Data Science Research, Research Organization of Information and Systems, Tachikawa 190-8518, Japan. ${ }^{\square}$ email: miyoshi@ isee.nagoya-u.ac.jp 


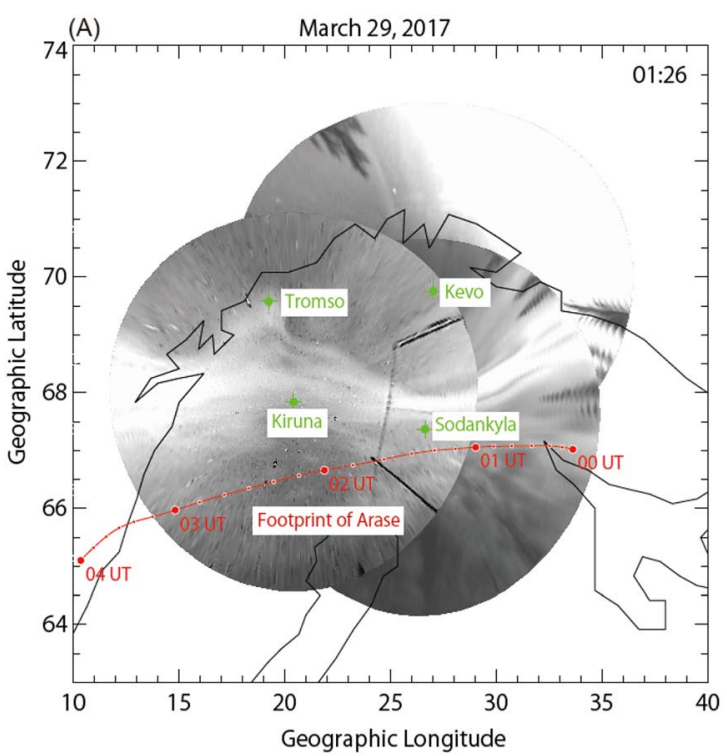

(B)

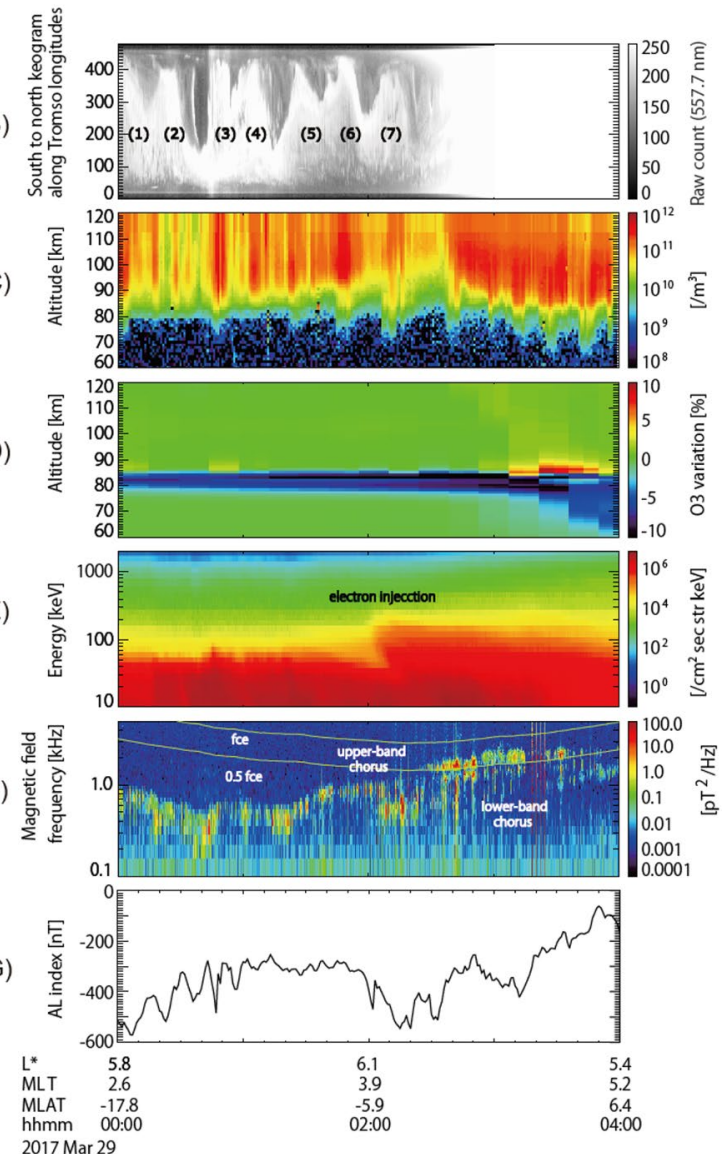

Figure 1. Geospace conditions from the upper atmosphere to the magnetosphere. (A) Map around the Scandinavian peninsula with the footprint of Arase from 00:00 UTC to 04:00 UTC on March 29, 2017. Aurora images were obtained from the all sky imager network. (B) Keograms of an aurora image along the longitudes of Tromsø, Norway. Labels (1-7) show the "omega-band" structures. (C) Electron density profile from a vertical beam of the EISCAT VHF radar at Tromsø, Norway, on March 29, 2017. The horizontal axis represents the universal time; the vertical axis denotes the altitude. The colour bar indicates the electron density. (D) Relative $\mathrm{O}_{3}$ profile from the computer simulation based on the EISCAT observations. The colour bar indicates relative variations from the controlled run without energetic electron precipitations. (E) Energy-time diagram of electrons measured by the MEP-e/HEP/XEP instruments onboard the Arase spacecraft. The horizontal axis represents the universal time; the vertical axis denotes the electron energy. Here, MEP-e, HEP, and XEP denote the medium-energy particle-electron, high-energy particle, and extremely high-energy particle, respectively. The colour bar indicates the differential flux of electrons. (F) Frequency-time diagram of the magnetic field components of plasma waves measured by the Arase spacecraft. The vertical axis denotes the plasma wave frequency. The colour bar indicates the power spectrum density of the waves. Two lines correspond to the electron gyrofrequency $\left(f_{c e}\right)$ and their hall frequency $\left(0.5 f_{e}\right)$. (G) AL index. (IDL ver.8.7, https://www.l3harrisge ospatial.com/Software-Technology/IDL).

The Van Allen radiation belt possesses the highest energy charged particle population in geospace, and relativistic electrons are trapped in the outer Van Allen radiation belt ${ }^{10,11}$. Precipitations of relativistic electron of the outer Van Allen radiation belt have been observed, which are categorized into different groups ${ }^{12,13}$. Relativistic electron microbursts ${ }^{14}$ that are spikes of high precipitation flux on subsecond timescales are observed. Recently, it has been suggested that relativistic electron microbursts occur during PsA and relativistic electrons precipitate into the upper and middle atmosphere ${ }^{15}$. The precipitating relativistic electrons can reach altitudes below the typical aurora emission altitudes, resulting in mesospheric ionization and the consequent depletion of ozone molecule $\mathrm{O}_{3}$ at mesospheric altitudes. However, previous conjugate satellite and ground-based optical observations ${ }^{15}$ have not detected such low-altitude ionization, so that the maximum energy of precipitating electrons is not known. The presence of high-energy electrons in PsA can only be inferred from direct observations of the electron density by incoherent scatter radars in the mesosphere in concert with ground-based optical imager and satellite observations. In the present study, we report unique coordinated experiments for the observation of PsA in Scandinavia, which were realized by the European Incoherent Scatter (EISCAT) radar $^{16}$, the all-sky camera network, and the Japanese spacecraft Arase ${ }^{17}$. The data are useful for estimating the possible ozone destruction due to relativistic electron precipitations associated with PsA. 

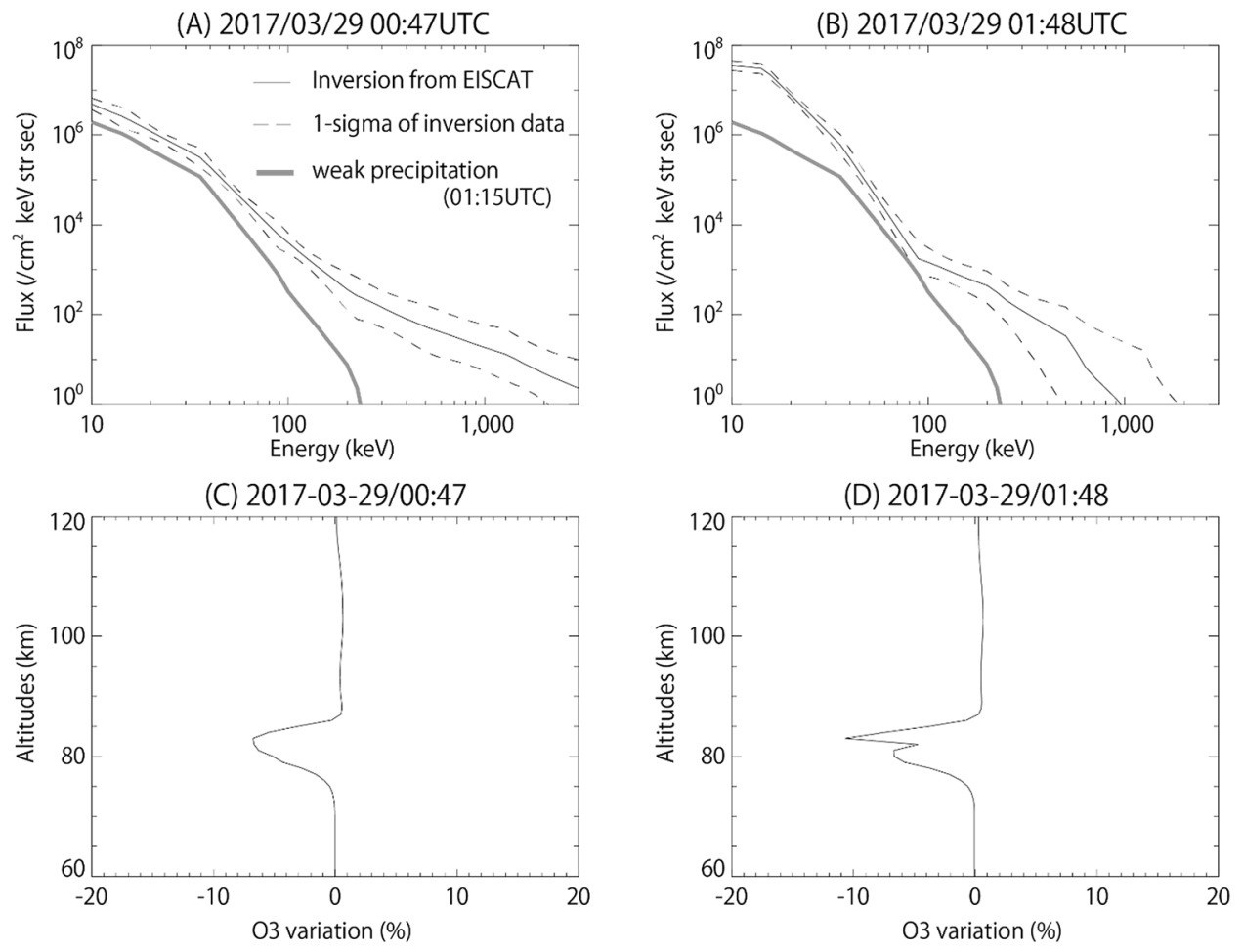

Figure 2. Energy spectrum of precipitating electrons and ozone depression. (A,B) Estimated energy spectrum obtained from EISCAT measurements using the inversion method ${ }^{12}$. The black dashed line indicates the 1-sigma errors. Thick line is a reference of weak electron precipitation observed at 01:15UTC on March 29, 2017. (C,D) Altitude profile of $\mathrm{O}_{3}$ variation. The profiles mean the relative difference to the control run without $\mathrm{MeV}$ electron precipitations.

\section{Arase and EISCAT observations}

At the end of March 2017, a high-speed solar wind stream arrived at Earth causing moderate geomagnetic storms for a few days. Figure $1 \mathrm{~A}$ illustrates the footprints of Arase mapped along the field line and presents aurora images obtained from the imager network. The Arase footprints traversed the Scandinavia Peninsula from 00:00 to 04:30 UTC on March 29,2017. The aurora image is a snapshot at 01:27 UTC, and wave-like structures, known as the "omega-band" aurora signature, are seen at $68^{\circ}-70^{\circ}$ latitudes. Figure $1 \mathrm{G}$ shows the AL index, from which several substorm activities are identified. The EISCAT radar at Troms $ø$, Norway, observed the ionospheric electron density profile at altitudes between 60 and $120 \mathrm{~km}$, which are directly related to the energy spectrum of precipitating electrons. The Arase spacecraft observed electrons and ions as well as electric and magnetic fields and waves in the Van Allen radiation belts.

Figures 1B-F illustrates a series of data obtained from the ground (aurora), ionosphere (electron precipitation), mesospheric ozone simulation, and magnetosphere. Figure $1 \mathrm{~B}$ shows an aurora keogram at the Troms $\varnothing$ longitude during the event period. A series of omega-band structures is developed every $30 \mathrm{~min}$; seven omegabands were identified in total, as shown in Fig. 1B. The vertical stripes appearing over a wide latitudinal range manifest the appearance of PsA. Figure 1C presents the temporal variation of the height profile of the electron density. The electron density enhancements occurred intermittently at altitudes below $70 \mathrm{~km}$. It is noteworthy that the electron density enhancements are seen at around $65 \mathrm{~km}$ after 03:00 UTC. The altitude of $65 \mathrm{~km}$ is one of the lowest observed ionization altitudes associated with PsA.

The energy spectrum of precipitating electrons is derived by an inversion calculation using the height profile of electron density ${ }^{18}$. Figure $2 A, B$ present the estimated energy spectrum of precipitating electrons obtained from the EISCAT observations at the selected time interval. Figure $2 \mathrm{~A}, \mathrm{~B}$ represent the spectra at 0:47 and 1:48 UTC, respectively, when the omega-band structures traversed above Tromsø, as shown at (3) and (6) in Fig. 1B. By comparing the time variations of the energy spectrum and keogram, we deduce that $\mathrm{MeV}$ electron precipitations occur in association with PsA embedded in the omega-bands, indicating that $\mathrm{MeV}$ electron precipitations seemingly correlate with the repeated development of the omega-bands. The maximum energy of precipitating electrons exceeds $2 \mathrm{MeV}$.

The ionization induced by such precipitations may cause chemical consequences, especially in the concentration of odd nitrogen $\left(\mathrm{NO}_{\mathrm{x}}=\mathrm{N}+\mathrm{NO}+\mathrm{NO}_{2}\right)$ via the dissociation of molecular nitrogen and odd hydrogen $\left(\mathrm{HO}_{\mathrm{x}}=\mathrm{H}+\mathrm{OH}+\mathrm{HO}_{2}\right)$ due to ion-pair production ${ }^{19}$, which can catalytically deplete mesospheric odd oxygen $\left(\mathrm{O}_{\mathrm{x}}=\mathrm{O}+\mathrm{O}_{3}\right)^{18}$. This scenario has been verified by computer simulations, including a comprehensive description of the ion chemistry at altitudes between 20 and $150 \mathrm{~km}^{18}$. 

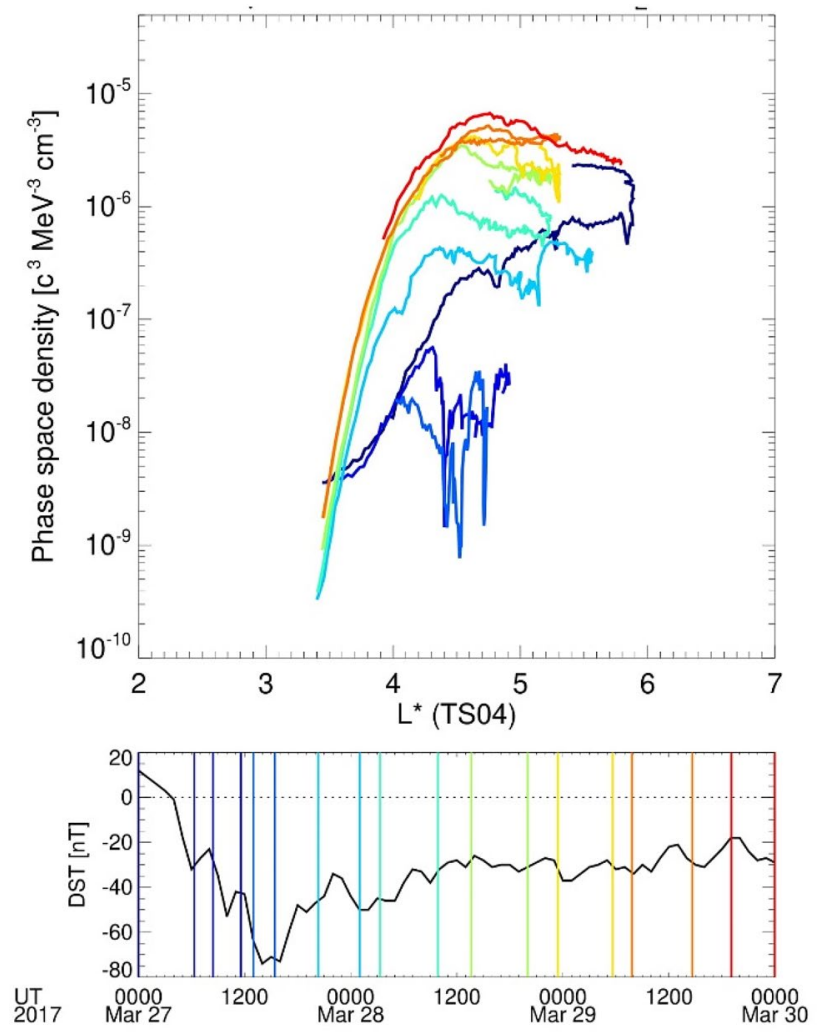

Figure 3. Evolutions of the phase space density of the outer belt during this interval (Top). Phase space density profiles measured by the XEP instrument ${ }^{15}$ on 27, 28, and 29 March 2017. Phase space density, $f$, is in units of $(\mathrm{c} / \mathrm{cm} \mathrm{MeV})^{3}$, where $c$ is the speed of light. By plotting phase space density at fixed magnetic adiabatic invariants, $\mu=1000 \mathrm{MeV} / \mathrm{G}$, and $\mathrm{K}=0.2 \mathrm{R}_{\mathrm{E}} \mathrm{G}^{1 / 2}$. The invariants were calculated using the TS04 magnetic field model ${ }^{49}$. Corresponding energy and pitch angle for $\mu=1000 \mathrm{MeV} / \mathrm{G}$, and $\mathrm{K}=0.2 \mathrm{R}_{\mathrm{E}} \mathrm{G}^{1 / 2}$ are shown in Fig. S1 (Bottom). The storm index Dst. Colour lines in top panel correspond to each period in bottom panel.

Figure 1D presents the temporal variation of the height profile of $\mathrm{O}_{3}$ concentration predicted by the computer simulation, Sodankylä Ion and Neutral Chemistry Model (SIC) ${ }^{20}$. The figure shows the relative variations of $\mathrm{O}_{3}$ between the cases with and without (control run) electron forcing based on the EISCAT measurements ${ }^{16}$. Above $80 \mathrm{~km}$, catalytic ozone depletion is inefficient due to lack of HOx production. On the other hand, the catalytic reaction sequences that cause ozone depletion require atomic oxygen which at night is abundant in the upper mesosphere only. Thus before 03:00 UTC, more than $10 \% \mathrm{O}_{3}$ depletion is predicted but around $80 \mathrm{~km}$ altitudes only, although strong precipitation is observed, as shown in Fig. 1C. After 03:00 UTC, solar UV radiation increases production of atomic oxygen throughout the mesosphere after the sunrise, and catalytic $\mathrm{O}_{3}$ depletion extends down to $60 \mathrm{~km}$ altitudes. Note that the short-term enhancement around $85 \mathrm{~km}$ is due to a combination of PsA-driven atomic oxygen production and lack of catalytic loss. Figure 2C,D show the height profile of $\mathrm{O}_{3}$ concentration at 0:47 and 1:48 UTC, respectively. The simulation results confirm that the largest $\mathrm{O}_{3}$ depletion of $10 \%$ is observed during the PsA. In particular, electron precipitation associated with PsA makes a dominant role in the production of $\mathrm{NO}_{\mathrm{x}}$ and $\mathrm{HO}_{\mathrm{x}}$, which leads to $\mathrm{O}_{3}$ depletion in the mesosphere.

Figure $1 \mathrm{E}$ shows the energy spectra of electrons trapped in the magnetosphere with energies from $7 \mathrm{keV}$ to $3 \mathrm{MeV}$ as a function of time; the data are acquired from multi-instrument measurements onboard the Arase spacecraft ${ }^{17,21-24}$. During the observation period, the Arase spacecraft observed the trapped $\mathrm{MeV}$ electrons of the outer Van Allen radiation belt. At around 02:00 UTC, a flux enhancement of electrons is observed above $100 \mathrm{keV}$ followed by subsequent enhancements of electron fluxes of tens of keV. This flux enhancement is referred to as electron injection, i.e., fresh electrons from the night-side plasma sheet enter the inner magnetosphere. These electrons are responsible for generating whistler-mode chorus waves ${ }^{25}$.

Figure $1 \mathrm{~F}$ shows the wave power of plasma waves as a function of the frequency and time observed by the Arase spacecraft ${ }^{17}$ during the above-mentioned period. Intense lower-band chorus (LBC) and upper-band chorus (UBC) waves are recorded below and above half the electron-gyrofrequency, respectively. The average amplitudes of the LBC and UBC waves at 02:30 UTC, when the Arase was located near the magnetic equator, are 60 pT and 80 pT, respectively, which are typical chorus wave amplitudes during $\mathrm{PsA}^{7}$. The ambient plasma density is $\sim 0.6 \mathrm{~cm}^{-3}$, which is estimated from the frequency of the upper-hybrid resonance waves ${ }^{26}$ and the ambient magnetic field ${ }^{24}$ measured by the Arase spacecraft.

The chorus waves are primarily responsible for the local acceleration of electrons in the Van Allen radiation belt, leading to a peak in the radial profile of the electron phase space density (PSD). A recent study ${ }^{27}$ reported 


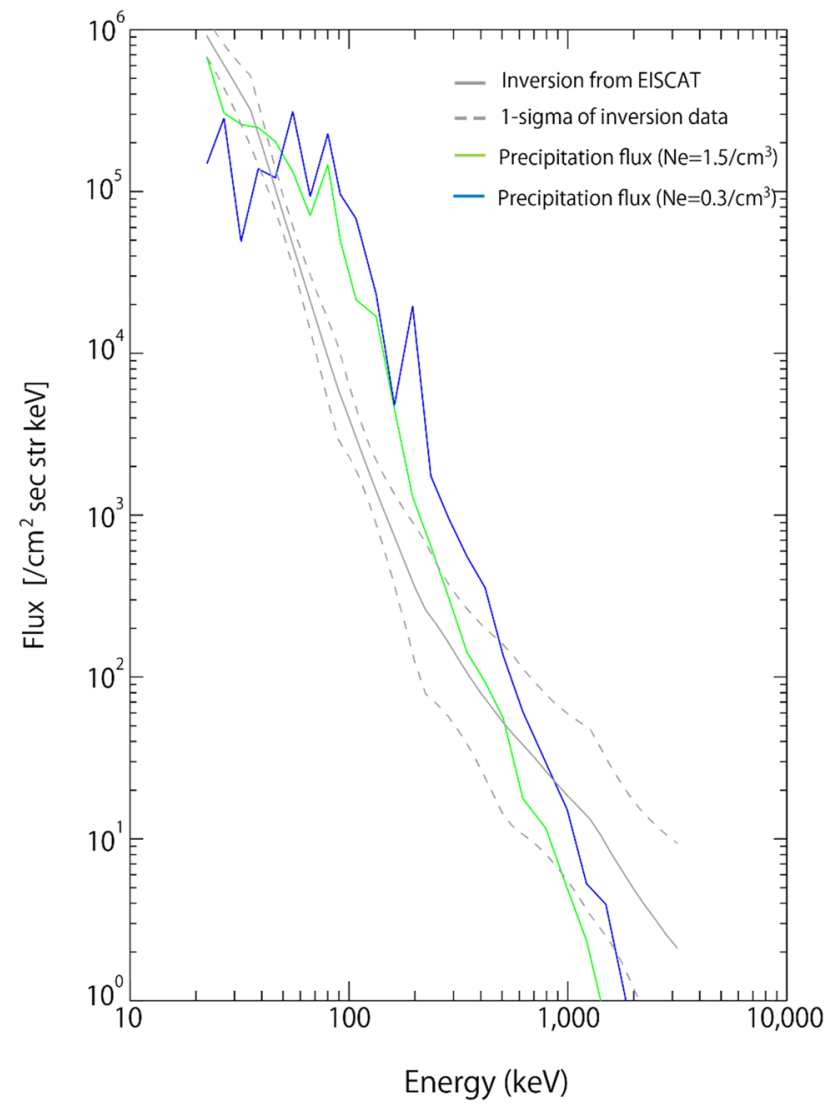

Figure 4. Comparison of wave-particle interaction simulation with EISCAT observations. Energy spectrum of precipitating electrons. The dark solid line indicates the energy spectrum obtained from EISCAT measurements using the inversion method ${ }^{14}$. The black dotted line indicates the 1-sigma errors. The blue and green solid lines indicate the energy spectrum obtained from the computer simulation ${ }^{21}$ of wave-particle interactions for an ambient density of $0.3 / \mathrm{cm}^{3}$ and $1.5 / \mathrm{cm}^{3}$, respectively.

the simultaneous acceleration and precipitation of $\mathrm{MeV}$ electrons owing to chorus waves. In fact, the radial profiles of the PSD at $1000 \mathrm{MeV} / \mathrm{G}$ and $0.2 \mathrm{R}_{\mathrm{E}} \mathrm{G}^{1 / 2}$ show a growing peak inside the Van Allen radiation belt during the storm, as shown in Fig. 3, suggesting that the chorus waves contribute to the local acceleration of Van Allen belt electrons ${ }^{28}$. Figure $\mathrm{S} 1$ shows corresponding energy and pitch angle for $1,000 \mathrm{MeV} / \mathrm{G}$ and $0.2 \mathrm{R}_{\mathrm{E}} \mathrm{G}^{1 / 2}$ along the satellite orbit.

\section{Discussion and summary}

Previous theoretical studies have suggested that chorus waves propagating towards higher latitudes can also produce electron precipitations over a wide energy range ${ }^{9,15,29,30}$. In this respect, we quantitatively estimate energy spectra of precipitating electron flux caused by chorus waves using a simulation of wave-particle interactions $\mathrm{s}^{31}$. We injected $2 \times 10^{6}$ test electrons along the field line and solved the equation of motion for each test electron. The equatorial flux distribution from $10 \mathrm{keV}$ to approximately $4 \mathrm{MeV}$ near the loss cone was determined to match that observed by Arase. We also computed the propagation of chorus waves by considering the Arase observed frequency spectrum. The wave amplitude used in the simulation was $80 \mathrm{pT}$ as an average during this interval, while the observed wave amplitude varied with time during this interval. We assumed that the plasma density observed by Arase remained constant along the magnetic field line and that the chorus waves were confined at latitudes below $40^{\circ}$, in agreement with statistical studies ${ }^{32}$.

Considering the uncertainty in the observed plasma density, we simulated two cases, as shown in Fig. 4, for $0.3 / \mathrm{cm}^{3}$ (blue solid line) and $1.5 / \mathrm{cm}^{3}$ (green solid line). The figure illustrates the estimated precipitation flux at the ionospheric altitudes and the electron spectrum derived from the inversion calculation of the EISCAT data. The dot-dashed lines indicate the 1-sigma error of the energy spectrum derived from EISCAT. During this interval, the chorus wave intensity varies with time, and intense chorus waves exceeding $200 \mathrm{pT}$ in amplitude are often observed. Moreover, the electron flux near the loss cone also varies with time; therefore, the energy spectrum from the simulation should exhibit temporal variation. The consistency between the simulation and the inversion calculation of the EISCAT data indicates that the observed chorus waves do cause the MeV electron precipitations during this interval. There are several discrepancies between the simulation and the EISCAT data. For example, the simulated flux of $30-80 \mathrm{keV}$ electrons is larger than that in the EISCAT data. The simulation assumes the uniform plasma density along the field-line, wave normal angles, which change the resonance 


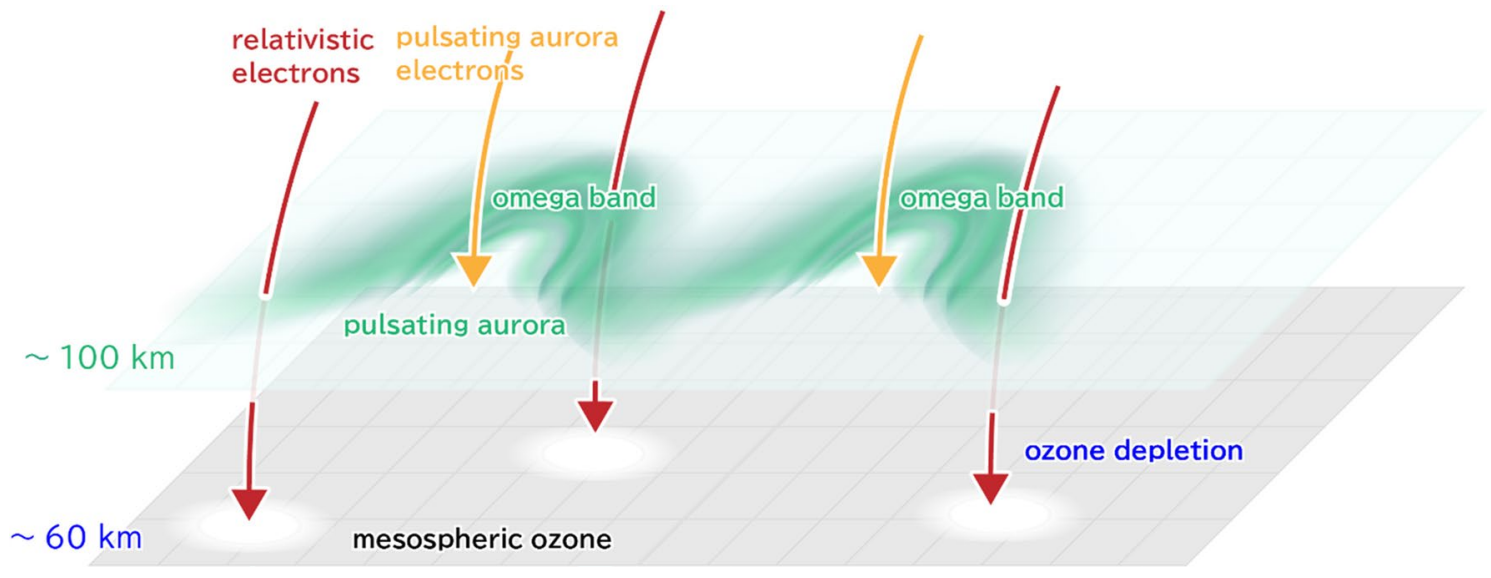

Figure 5. Schematic figure about simultaneous precipitations of tens $\mathrm{keV}$ electrons and relativistic electron precipitations. Schematic figure shows tens of $\mathrm{keV}$ electrons that cause pulsating aurora near the omega bands at the lower thermosphere. Simultaneously, sub-relativistic/relativistic electrons precipitate further into the mesospheric altitudes, causing significant ozone depletion. (Adobe Illustrator cc 2019. https://www.adobe.com// products/illustrator.html).

conditions, scattering rate, and the propagation latitudes. More accurate parameters of waves and electron flux are essential for future comparisons with the EISCAT data. Electro-Magnetic Ion Cyclotron (EMIC) waves can also cause $\mathrm{MeV}$ electron scattering. However, during this interval, the Arase spacecraft measurements showed no evidence of EMIC waves; we can therefore focus on interactions with chorus waves ${ }^{33}$.

Figure 5 shows a schematic diagram illustrating the simultaneous precipitation of $10 \mathrm{~s}$ of keV electrons, which reach the lower thermosphere, and $100 \mathrm{~s}$ of $\mathrm{keV}$ to $\mathrm{MeV}$ electrons penetrating even deeper into the mesospheric altitudes. They rush toward the Earth along the same field lines: the former brightens PsA, while the latter causes a local concentration change in mesospheric $\mathrm{O}_{3}$, as demonstrated by this study. In the upper mesosphere, the impact is similar to the quantitatively well-known effect of solar proton events ${ }^{19}$. Since PsA occur more frequently (almost daily) than well-known effect of solar proton events ${ }^{20}$, last much longer than our simulated single event, and often extend over large areas ${ }^{3}$, we expect the consequent variations in the $\mathrm{O}_{3}$ destruction to be significant. Previous studies ${ }^{34,35}$ frequently observed PsA events with a duration of $9 \mathrm{~h}$ or even longer, suggesting that mesospheric $\mathrm{O}_{3}$ depletion occurs in a wide magnetic local time. Therefore, the PsA effect must be considered when investigating the long-term composition variations of the middle and upper atmosphere, which manifests the multidisciplinary nature of the interaction between these atmospheric layers and the magnetosphere. In the future, our calculated PsA-driven $\mathrm{O}_{3}$ variations should be verified by conducting observations with, e.g., ground-based millimeter-wave spectroscopic radiometers ${ }^{36}$. In comparisons with satellite-based observations statistical methods can also be used to reveal both the short and longer-term ozone responses to PsA forcing ${ }^{37}$.

\section{Experimental methods}

Test-particle simulation. We used the geospace environment modelling system for integrated studiesradiation belt with wave-particle interaction module test particle simulation ${ }^{38}$, which simulates the wave-particle interaction process between lower band chorus propagating along the field line and the bouncing electrons. The number of test particle is $2 \times 10^{6}$, which are distributed from $10 \mathrm{keV}$ to $3 \mathrm{MeV}$ at the equatorial pitch angle range from $3^{\circ}$ to $30^{\circ}$. The simulation estimates the temporal variation of the energy of the precipitating electrons at an altitude of $100 \mathrm{~km}$. The electron momentum changes associated with the wave-particle interaction are given by the following equation of motion:

$$
\frac{d}{d t} p_{e}=q\left(\delta E+v_{e} \times(B+\delta B)\right)
$$

where $v_{e}=p_{e} / m_{e} \gamma$ is the electron velocity, $B$ is the background magnetic field vector, $p_{e}$ is the electron momentum, $q$ is the charge of an electron, $m_{e}$ is the electron rest mass, $\gamma$ is the Lorentz factor, and $\delta E$ and $\delta B$ are the electric and magnetic field perturbations that satisfy the dispersion relation of the parallel propagating whistler mode wave. When electrons interact with the waves, the equation of motion is numerically solved with the time step $\delta t$ during $\Delta t$, where $\delta t$ is chosen to resolve the gyromotion and $\Delta t$ is the time step chosen to solve the adiabatic guiding center motion. After calculation of the momentum change in $\Delta t$, the first adiabatic invariant of the electron at $t+\Delta t$ is calculated using the background magnetic field intensity at the electron position. Simultaneously with the scattering process, the electron guiding center position is advanced, in keeping with the first and second adiabatic invariants. The perturbation components are the same as that reported in previous study $^{29}$. The minimum frequency and the maximum frequencies are $0.3 f_{c e q}$, where $f_{c e q}$ is the electron cyclotron frequency at the magnetic equator and $0.5 f_{c e q}$, respectively. The duration of each element is $100 \mathrm{~ms}$ and sweep rate of each element is $2.0 f_{c e q}$. The bursts appear every $5 \mathrm{~s}$, and three rising tone elements are embedded in each burst. The repeat frequency of the rising tone elements is $3 \mathrm{~Hz}$, which is a typical modulation frequency of the internal modulation of PsA ${ }^{15}$. 
Ion-chemistry simulation at the upper/middle atmosphere. We used the Sodankylä Ion and Neutral Chemistry (SIC) model that is a 1-D atmospheric model which solves for concentration of 16 minor neutral species (including $\mathrm{HOx}, \mathrm{NOx}$, and $\mathrm{Ox}$ ) and 72 ion species at altitudes between 20 to $150 \mathrm{~km}^{20}$. The model includes 389 ion-neutral and neutral-neutral reactions, 2523 ion-ion and electron-ion recombination reactions, and molecular and eddy diffusion. The background neutral atmosphere (for example, $\mathrm{N}_{2}, \mathrm{O}_{2}$, and temperature) are calculated using the empirical NRLMSISE-00 model which depends on daily average values of solar F10.7 radio flux and geomagnetic activity through the Ap index. The daily average solar spectrum is calculated using the SOLAR2000 empirical solar irradiance model. In addition to solar radiation, SIC can be driven by electron precipitations, which has been used in this study. A detailed description of the SIC model is given in ${ }^{20}$.

Estimation of energetic electron spectrum from EISCAT observations. We used the inversion method by utilizing a Metropolis-Hastings Markov Chain Monte Carlo method (MCMC) and the SIC model as a forward theory of the ionospheric response to the precipitations. The detail procedure of the inversion is described in ${ }^{18}$.

\section{Data availability}

The Arase data is available from the ERG Science Centre operated by ISAS/JAXA and ISEE/Nagoya University (https://ergsc.isee.nagoya-u.ac.jp/data_info/index.shtml.en) ${ }^{39}$. The present data analysed the MEP e L2 v01_02 $2^{40,41}$, HEP L2 v03_01 ${ }^{42,43}$, XEP L2 v01_00 ${ }^{44}$, PWE/OFA L2 v02_01 ${ }^{45}$, MGF L2 v03_04 ${ }^{46}$, Orbit L3 v01 data ${ }^{47}$. The ground-based optical data from Kiruna, Sweden, used in this paper were obtained through the database of optical instruments at the National Institute of Polar Research, Japan (http://pc115.seg20.nipr.ac.jp/www/opt/). The EISCAT data used in this paper were obtained through the database of EISCAT at the National Institute of Polar Research, Japan (http://polaris.nipr.ac.jp/ eiscat/eiscatdata/). The Dst index data and the AL index were provided by the World Data Centre for Geomagnetism, Kyoto (http://wdc.kugi.kyoto-u.ac.jp/wdc/Sec3.html) ${ }^{48}$.

Received: 16 March 2021; Accepted: 14 June 2021

Published online: 13 July 2021

\section{References}

1. Akasofu, S. I. The development of the auroral substorm. Planet Space Sci. 4, 273-282 (1964).

2. Meng, C.-I., Mauk, B. \& McIlwain, C. E. Electron precipitation of evening diffuse aurora and its conjugate electron fluxes near the magnetic equator. J. Geophys. Res. 84, 2545. https://doi.org/10.1029/JA084iA06p02545 (1979).

3. Lessard, M. R. A review of pulsating aurora. In Auroral Phenomenology and Magnetospheric Processes: Earth and Other Planets, Geophysical Monograph Series Vol. 197 (ed. Keiling, A.) (Springer, 2012).

4. Miyoshi, Y. et al. Time of flight analysis of pulsating aurora electrons, considering wave-particle interactions with propagating whistler mode waves. J. Geophys. Res. 115, A10312 (2010).

5. Sandahl, I., Eliasson, L. \& Lundin, R. Rocket observations of precipitating electrons over a pulsating aurora. Geophys. Res. Lett. 7(5), 309-312 (1980).

6. Yau, A. W., Whalen, B. A. \& McEwen, D. J. Rocket-borne measurements of particle pulsation in pulsating aurora. J. Geophys. Res. 86, 5673-5681 (1980).

7. Nishimura, Y. et al. Identifying the driver of pulsating aurora. Science 330, 81-84 (2010).

8. Kasahara, S. et al. Pulsating aurora from electron scattering by chorus waves. Nature 554, 337-340 (2018).

9. Grandin, M. et al. Observation of pulsating aurora signatures in cosmic noise absorption data. Geophys. Res. Lett. 44, 5292-5300. https://doi.org/10.1002/2017GL073901 (2017).

10. Li, W. \& Hudson, M. K. Earth's Van Allen Radiation Belts: From discovery to the Van Allen Probes Era. J. Geophys Res. 124, 8319-8351. https://doi.org/10.1029/2018JA025940 (2019).

11. Thorne, R. M. et al. Rapid local acceleration of relativistic radiation belt electrons by magnetospheric chorus. Nature 504, 411-414 (2013).

12. Vampola, A. L., Koons, H. C. \& McPherson, D. A. Outer-zone electron precipitation. J. Geophys. Res. 76, 7609-7617. https://doi. org/10.1029/JA076i031p07609 (1971).

13. Blum, L. W. et al. Rapid MeV electron precipitation as observed by SAMPEX/HILT during high-speed stream driven storms. J. Geophys. Res. 120, 3783-3794. https://doi.org/10.1002/2014JA020633 (2015).

14. Imhof, W. L. et al. Relativistic electron microbursts. J. Geophys. Res. 97, 13829-13837. https://doi.org/10.1029/92JA01138 (1992).

15. Miyoshi, Y. et al. Energetic electron precipitation associated with pulsating aurora: EISCAT and Van Allen Probes observations. J. Geophys. Res. 120, 2754-2766. https://doi.org/10.1002/2014JA020690 (2015).

16. Rishbeth, H. \& Williams, P. J. S. The EISCAT ionospheric radar: The system and its early results. Q. J. R. Astron. Soc. 26, 478-512 (1985).

17. Miyoshi, Y. et al. Geospace Exploration Project ERG. Earth Planets Space 70, 101 (2018).

18. Turunen, E. et al. Mesospheric ozone destruction by high-energy electron precipitation associated with pulsating aurora. J. Geophys. Res. Atmos. 121, 11852-11861 (2016).

19. Verronen, P. T. \& Lehmann, R. Analysis and parameterization of ionic reactions affecting middle atmospheric HOx and NOy during solar proton events. Ann. Geophys. 31, 909-956 (2013).

20. Verronen, P. T. et al. Diurnal variation of ozone depletion during the October-November 2003 solar proton events. J. Geophys. Res. 110, A09S32. https://doi.org/10.1029/2004JA010932 (2005).

21. Kasahara, S. et al. Medium-energy particle experiments-electron analyzer (MEP-e) for the exploration of energization and radiation in geospace (ERG) mission. Earth Planets Space 70, 69 (2018).

22. Mitani, T., Takashima, T., Kasahara, S., Miyake, W. \& Hirahara, M. High-energy electron experiments (HEP) aboard the ERG (Arase) satellite. Earth Planets Space 70, 77 (2018).

23. Higashio, N., Takashima, T., Shinohara, I. \& Matsumoto, H. The extremely high-energy electron experiment (XEP) onboard the Arase (ERG) satellite. Earth Planets Space 70, 134 (2018).

24. Kasahara, Y. et al. The plasma wave experiment (PWE) on board the Arase (ERG) satellite. Earth Planets Space 70, 86 (2018).

25. Jaynes, A. et al. Source and seed populations for relativistic electrons: Their roles in radiation belt chances. J. Geophys. Res. 120, 7240. https://doi.org/10.1002/2015JA021234 (2015).

26. Kumamoto, A. et al. High frequency analyzer (HFA) of plasma wave experiment (PWE) onboard the Arase spacecraft. Earth Planets Space 70, 82 (2018). 
27. Kurita, S., Miyoshi, Y., Blake, B., Reeves, G. \& Kletzing, C. Relativistic electron microbursts and variations in trapped MeV electron fluxes during the 8-9 October 2012 storm: SAMPEX and Van Allen probes observations. Geophys. Res. Lett. 43, 3017-3025. https:// doi.org/10.1002/2016GL068260 (2016).

28. Reeves, G. D. et al. Electron acceleration in the heart of the Van Allen radiation belts. Science 341, 991-994 (2013).

29. Miyoshi, Y. et al. Relativistic electron microbursts as high-energy tail of pulsating aurora electrons. Geophys. Res. Lett. 47, e2020GL090360. https://doi.org/10.1029/2020GL090360 (2020).

30. Wang, D. \& Shprits, Y. Y. On how high-latitude chorus waves tip the balance between acceleration and loss of relativistic electrons. Geophys. Res. Lett. 46(14), 7945-7954. https://doi.org/10.1029/2019GL082681 (2019).

31. Saito, S., Miyoshi, Y. \& Seki, K. Relativistic electron microbursts associated with whistler chorus rising tone elements: GEMSISRBW simulations. J. Geophys. Res. 117, A10206. https://doi.org/10.1029/2012JA018020 (2012).

32. Santolík, O., Macúšová, E., Kolmašová, I., Cornilleau-Wehrlin, N. \& de Conchy, Y. Propagation of lower-band whistler-mode waves in the outer Van Allen belt: Systematic analysis of 11 years of multi-component data from the Cluster spacecraft. Geophys. Res. Lett. 41, 2729-2737. https://doi.org/10.1002/2014GL059815 (2014).

33. Miyoshi, Y. et al. Precipitation of radiation belt electrons by EMIC waves, observed from ground and space. Geophys. Res. Lett. 35, L23101. https://doi.org/10.1029/2008GL035727 (2008).

34. Jones, S. L., Lessard, M. R., Rychert, K., Spanswick, E. \& Donovan, E. Large scale aspects and temporal evolution of pulsating aurora. J. Geophys. Res. 116, A03214 (2011).

35. Jones, S. L. et al. Persistent widespread pulsating aurora: A case study. J. Geophys. Res. 118, 2998-3006 (2013).

36. Isono, Y. et al. Ground-based observations of nitric oxide in the mesosphere and lower thermosphere over Antarctica in 2012-2013. J. Geophys. Res. 119, 7745-7761 (2014).

37. Andersson, M. E., Verronen, P. T., Rodger, C. J., Clilverd, M. A. \& Seppälä, A. Missing driver in the Sun-Earth connection from energetic electron precipitation impacts mesospheric ozone. Nat. Commun. 5, 5197. https://doi.org/10.1038/ncomms6197 (2014).

38. Matsuoka, A. et al. The ARASE (ERG) magnetic field investigation. Earth Planet Space 70, 43 (2018).

39. Miyoshi, Y. et al. The ERG Science Center. Earth Planets Space 70, 96 (2018).

40. Kasahara, S. et al. The MEP-e Instrument Level-2 3-D Flux Data of Exploration of Energization and Radiation in Geospace (ERG) Arase Satellite (ERG Science Center, 2018).

41. Kasahara, S. et al. The MEP-e Instrument Level-2 Omni-Directional Flux Data of Exploration of Energization and Radiation in Geospace (ERG) Arase Satellite (ERG Science Center, 2018).

42. Mitani, T. et al. The HEP instrument Level-2 3-D Flux Data of Exploration of Energization and Radiation in Geospace (ERG) Arase Satellite (ERG Science Center, 2018).

43. Mitani, T. et al. The HEP Instrument Level-2 Omni-Directional Flux Data of Exploration of Energization and Radiation in Geospace (ERG) Arase Satellite (ERG Science Center, 2018).

44. Higashio, N. et al. The XEP Instrument Level-2 Omni Flux Data of Exploration of Energization and Radiation in Geospace (ERG) Arase Satellite (ERG Science Center, 2018).

45. Kasahara, Y. et al. The PWE/OFA Instrument Level-2 Spectrum Data of Exploration of Energization and Radiation in Geospace (ERG) Arase Satellite (ERG Science Center, 2018).

46. Matsuoka, A. et al. The MGF Instrument Level-2 Spin Fit Magnetic Field Data of Exploration of Energization and Radiation in Geospace (ERG) Arase Satellite (ERG Science Center, 2018).

47. Miyoshi, Y., Shinohara, I. \& Jun, C.-W. The Level-3 Orbit Data of Exploration of Energization and Radiation in Geospace (ERG) Arase Satellite (ERG Science Center, 2018).

48. Tsyganenko, N. A. \& Sitnov, M. I. Modelling the dynamics of the inner magnetosphere during strong geomagnetic storms. J. Geophys. Res. 110, A03208 (2005).

49. Nose, M., Iyemori, T., Sugiura, M. \& Kamei, T. Geomagnetic Dst Index (World Data Center for Geomagnetism, 2015).

\section{Acknowledgements}

We thank the entire exploration of energization and radiation in geospace (ERG) project team for the support in realizing the observations. We are indebted to the director and staff of EISCAT for operating the facility and supplying the data. EISCAT is an international association supported by China (CRIRP), Finland (SA), Germany (DFG), Japan (NIPR and ISEE, Nagoya), Norway (NFR), Sweden (VR), and the United Kingdom (PPARC).

\section{Author contributions}

Y.M. conceived and designed the study, analysed the data, and wrote the initial draft. K.H. developed the groundbased optical instruments used in this study together with Y.M., S.O., Y.O., and S.K. K.H. oversaw the production of the data sets and discussed its interpretation. Y.O. processed the optical data from Kiruna and discussed the results. S.S. discussed the interpretation of the data. I.S. oversaw the ERG project and discussed the interpretation of the event. A.K., P.T.V., and E.T. conducted the simulation of ion chemistry at the middle atmosphere. S.K. and S.Y. led the development and operation of MEP and T.H., and K.K. contributed to the data processing. T.M. and T.T. led the development and operation of HEP, and N.H. led the development and operation of XEP. Y.K. led the development and operation of PWE with the contribution of S.M., A.K., and F.T. M.S. contributed to the processing of the PWE data. A.M. led the development and operation of MGF. A.T., S.I. and S. N. contributed to the data processing. C.J. contributed to the acquisition of the orbit data. All authors reviewed the manuscript.

\section{Funding}

This research was supported by Grants-in-Aid for Scientific Research (grant number 15H05747 to Y.M., K.H., S.K, S.O., S.S., and Y.O., grant number 16H06286 to Y.M., S.O, and I.O., grand number 17H06140, to I.S., and grand number $20 \mathrm{H} 01959$ to Y.M., S.S., and S.K.) of the Japan Society for the Promotion of Science (JSPS). The work of A.K was funded by the Tenure Track Project in Radio Science at the Sodankylä Geophysical Observatory/University of Oulu. The work of P.T.V. was supported by the Academy of Finland (project \#335555 ICT-SUNVAC).

\section{Competing interests}

The authors declare no competing interests. 


\section{Additional information}

Supplementary Information The online version contains supplementary material available at https://doi.org/ 10.1038/s41598-021-92611-3.

Correspondence and requests for materials should be addressed to Y.M.

Reprints and permissions information is available at www.nature.com/reprints.

Publisher's note Springer Nature remains neutral with regard to jurisdictional claims in published maps and institutional affiliations.

(c) (i) Open Access This article is licensed under a Creative Commons Attribution 4.0 International License, which permits use, sharing, adaptation, distribution and reproduction in any medium or format, as long as you give appropriate credit to the original author(s) and the source, provide a link to the Creative Commons licence, and indicate if changes were made. The images or other third party material in this article are included in the article's Creative Commons licence, unless indicated otherwise in a credit line to the material. If material is not included in the article's Creative Commons licence and your intended use is not permitted by statutory regulation or exceeds the permitted use, you will need to obtain permission directly from the copyright holder. To view a copy of this licence, visit http://creativecommons.org/licenses/by/4.0/.

(C) The Author(s) 2021 